\title{
X-ray Spectroscopic Studies of a Solid-Density Germanium Plasma Created by a Free Electron Laser
}

\author{
Gabriel Pérez-Callejo 1® ${ }^{\circledR}$, Sam M. Vinko ${ }^{1}{ }^{\circledR}$, Shenyuan Ren ${ }^{1}$, Ryan Royle ${ }^{1}$, \\ Oliver Humphries ${ }^{1,2}(\mathbb{D})$, Thomas R. Preston ${ }^{3}$ (D), Bruce A. Hammel ${ }^{4}$, Hyun-Kyung Chung ${ }^{5,6}$, \\ Tomas Burian ${ }^{7}$, Vojtěch Vozda ${ }^{7}$, Ming-Fu Lin ${ }^{8}$, Tim Brandt van Driel ${ }^{8}$ and Justin S. Wark ${ }^{1, *(D)}$ \\ 1 Clarendon Laboratory, Department of Physics, University of Oxford, Parks Road, Oxford OX1 3PU, UK; \\ gabriel.perezcallejo@physics.ox.ac.uk (G.P.-C.); sam.vinko@physics.ox.ac.uk (S.M.V.); \\ shenyuan.ren@physics.ox.ac.uk (S.R.); ryan.royle@physics.ox.ac.uk (R.R.); \\ oliver.humphries@physics.ox.ac.uk (O.H.) \\ 2 Helmholtz Zentrum Dresden Rossendorf, Bautzner Landstraße 400, 01328 Dresden, Germany \\ 3 European XFEL, Holzkoppel 4, 22869 Schenefeld, Germany; thomas.preston@xfel.eu \\ 4 Lawrence Livermore National Laboratory, Livermore, CA 94550, USA; bhammel@llnl.gov \\ 5 National Fusion Research Institute, Daejeon 34133, Korea; hkchung@nfri.re.kr \\ 6 Gwangju Institute of Science and Technology, Gwangju 61005, Korea \\ 7 Institute of Physics, Czech Academy of Sciences, Na Slovance 2, 18221 Prague 8, Czech Republic; \\ burian@fzu.cz (T.B.); vozda@fzu.cz (V.V.) \\ 8 SLAC National Accelerator Laboratory, 2575 Sand Hill Road, Menlo Park, CA 94025, USA; \\ mfucb@SLAC.Stanford.edu (M.-F.L.); timbvd@slac.stanford.edu (T.B.v.D.) \\ * Correspondence: justin.wark@physics.ox.ac.uk
}

Received: 21 October 2020; Accepted: 16 November 2020; Published: 18 November 2020

\begin{abstract}
The generation of solid-density plasmas in a controlled manner using an X-ray free electron laser (XFEL) has opened up the possibility of diagnosing the atomic properties of hot, strongly coupled systems in novel ways. Previous work has concentrated on K-shell emission spectroscopy of low $Z$ $(<=14)$ elements. Here, we extend these studies to the mid- $Z(=32)$ element Germanium, where the XFEL creates copious L-shell holes, and the plasma conditions are interrogated by recording of the associated L-shell X-ray emission spectra. Given the desirability of generating as uniform a plasma as possible, we present here a study of the effects of the FEL photon energy on the temperatures and electron densities created, and their uniformity in the FEL beam propagation direction. We show that good uniformity can be achieved by tuning the photon energy of the XFEL such that it does not overlap significantly with L-shell to M-shell bound-bound transitions, and lies below the L-edges of the ions formed during the heating process. Reasonable agreement between experiment and simulations is found for the emitted $X$-ray spectra, demonstrating that for these higher $Z$ elements, the selection of appropriate XFEL parameters is important for achieving uniformity in the plasma conditions.
\end{abstract}

Keywords: X-ray spectroscopy; germanium; solid density; LCLS

\section{Introduction}

In some of the first studies of the effects of focussing the output of the X-ray Free Electron Laser (XFEL) pulse of the Linear Coherent Light Source (LCLS) at irradiances of order $10^{17} \mathrm{Wcm}^{-2}$ onto foil targets, it was demonstrated that hot (up to $200 \mathrm{eV}$ ) plasmas could be created at exactly solid density [1,2]. The materials studied to date have been low- $\mathrm{Z}$ elements, such as $\mathrm{Mg}, \mathrm{Al}$, and $\mathrm{Si}$, with the incident photon energy of the XFEL being around and above the associated K-shell ionisation energies-i.e., in the 1-2 keV range. Diagnosis of the plasma conditions and associated physics was made by observing the K-shell emission of the various charge states produced, with L-shell electrons 
filling the single or double core holes in the ions created by the XFEL, thus leading, in many cases, to emission all the way up to the hydrogenic species (i.e., Ly- $\alpha$ ). As the final temperatures produced are insufficient to thermally excite the K-shell of such ions, the observed emission spectrum is gated by the pulse length of the LCLS beam itself (typically sub-100 fs), and on such a timescale, the micron scale solid density plasma is effectively confined by its own inertia.

Detailed studies of the X-ray spectra have led to an improved understanding of ionization potential depression (IPD) [3-5], as well as affording measurements of the femtosecond collisional ionization rates by means of the Auger-clock method [6,7]. Given the relatively long penetration depth of the X-rays compared with optical light, a study of the emission from slabs of plasma of varying thickness (from a few tens to a few hundred $\mathrm{nm}$ ) has allowed direct measurements of $\mathrm{K}$-shell opacity [8].

In addition to the above experiments, saturable absorption of the incident $\mathrm{X}$-ray pulse has also been demonstrated by tuning of the photon energy of the incident beam, such that when it creates and heats the solid-density sample, the K edges of the ions present in the hot collisional plasma-which have energies that increase as a function of ionization have energies exceeding the photon energy of the $\mathrm{X}$-rays [9]. Saturable absorption is a potentially useful phenomenon, in that it increases the effective absorption length of the X-rays, leading to more uniform deposition of the incident $\mathrm{X}$-ray energy, with a concomitant reduction in temperature and electron density gradients in the direction of the XFEL beam. Such an improvement in the uniformity of the plasma conditions is of importance in experiments designed to specifically measure plasma parameters as a function of temperature and electron density.

The X-ray spectra collected in the experiments mentioned above have been simulated by use of the atomic-kinetics code SCFLY [10]. SCFLY is a thoroughly revised version of the widely available collisional-radiative FLYCHK suite [11,12], and is specifically tailored for X-ray laser problems, using super-configurations to describe the atomic physics of the system [13]. In comparison with FLYCHK, rather than using a simple screened hydrogenic model, SCFLY uses calculated atomic quantities that are given as an input by the user generated, for example, by the DHS code [14]. However, despite its usefulness and evident successes, it should be borne in mind that SCLFY is a 0-D code, and as such, is not well-suited for modelling situations where the incident $\mathrm{X}$-rays are rapidly absorbed, resulting in significant gradients along the direction of incidence of the FEL beam. Although such effects can, to a certain extent, be accounted for by use of multiple simulations of successive thin laminae of plasma-with the calculated exit spectrum of the FEL from one lamina being used as the incident beam for the next [9], such an approach is far from ideal. We return to this point below, when we outline the use of the multi-dimensional Cretin code for the work presented in this paper [15].

It is in the above context that we present here the results of experiments and simulations of the heating of a solid sample of a medium atomic number material, Germanium $(Z=32)$, by the focused output of LCLS. As in the previous K-shell work mentioned briefly above, the Ge plasma was created by irradiation with relatively soft $\mathrm{X}$-rays, with energies ranging from 1300 to $1600 \mathrm{eV}$, just above the cold L-III edge, which lies at $1216 \mathrm{eV}$. As such, we expect that as the plasma is heated, it will pass through ion stages that will have L-edge energies of order or greater than the XFEL photon energy, impacting the total absorbed energy, and the uniformity of its deposition, akin to the way in which the $\mathrm{K}$-edges of the lower $Z$ elements shifted in those experiments. However, and in contrast to the cases where we studied the K-shell spectra of lower $Z$ elements, the Ge ions will also have a plethora of L-M bound-bound transitions at energies that could overlap those of the heating $X$-rays. Such bound transitions were largely absent in the lower $Z$ work, as the M shell electrons of the cold solid are already free for cold $\mathrm{Mg}$ and $\mathrm{Al}$, and the measured IPDs indicate that rebinding of the $\mathrm{M}$-shell would not have taken place for those elements, except perhaps at the highest ion stages. Given that both the relevant edge structures and bound-bound transitions of the various ion stages of Ge will alter the energy deposition of the FEL as the Ge plasma passes through the various ion stages as it is heated, we might thus expect a degree of richness and complexity in the way in which the intense LCLS beam interacts 
with this system: indeed this is the case, and in this energy range, we find that the greatest uniformity of energy deposition occurs for an incident photon energy around $1400 \mathrm{eV}$, with an associated lower overall electron temperature than obtained with LCLS photon energies immediately above and below this value, and we discuss below the underlying atomic physics underpinning this finding.

The paper is laid out in the following manner. In Section 2, we discuss the experimental set-up and materials used, as well as the different diagnostics that were fielded in the experiments. Additionally, the radiative transfer simulations used to model the results are described. Section 3 presents the results of these simulations, as well as those from experimental measurements. Special emphasis is placed on the computational predictions for the temperature and ionisation distribution along the targets. These results are compared with the experimental measurements, and their relation is discussed. Finally, a summary of the findings is presented.

\section{Materials and Methods}

The experiments were conducted at the Soft X-ray (SXR) endstation [16] of the LCLS. The X-ray pulse, which had an approximately Gaussian temporal envelope with a Full Width Half Maximum (FWHM) of $60 \mathrm{fs}$ [17], was focused on $508 \mathrm{~nm}$ thick germanium foils, mounted on a silicon substrate. The targets were oriented at an angle of $45^{\circ}$ with respect to the incident laser beam. The nominal energy of the laser was $\sim 2 \mathrm{~mJ}$, reduced to $0.7 \mathrm{~mJ}$ after transmission through the beamline optics [18]. The photon energy of the XFEL pulse was varied between 1300 and $1600 \mathrm{eV}$ in $100 \mathrm{eV}$ steps, with a nominal bandwidth of $0.4 \%$ of the central photon energy in all cases. The spatial profile of the XFEL spot was measured using imprint measurements on $\mathrm{PbI}_{2}$ targets mounted on a polymethyl methacrylate (PMMA) substrate [19]. The effective area of the laser spot was measured to be $28.5 \mathrm{\mu m}^{2}$, resulting in a peak intensity of the XFEL of $\sim 5 \times 10^{16} \mathrm{~W} \mathrm{~cm}^{-2}$. The measured spot profile is shown in Figure $1 \mathrm{~b}$, where the $y$-axis indicates the fluence normalised to its peak, and the $x$-axis indicates the area enclosed by the corresponding iso-fluence profile, as detailed in Ciricosta et al. [10].

$X$-ray spectroscopy was used to diagnose the plasma. The spectra of the emission at $30^{\circ}$ with respect to the target normal were measured with a flat-crystal Bragg spectrometer which used a Beryl $(10 \overline{1} 0)$ crystal $(2 d=15.96 \AA)$ for diffracting the X-rays before they were recorded by a Princeton Instruments CCD camera. The angular set-up of the experiment was designed so that the spectrometer was sensitive to X-rays between 1100 and $1600 \mathrm{eV}$, to observe the emission from L-shell transitions. A schematic drawing of the experimental set-up is shown in Figure 1a.

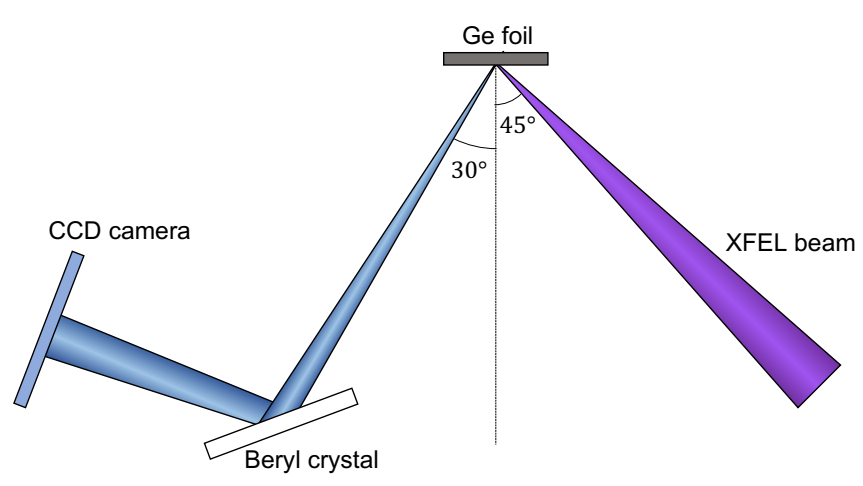

(a)

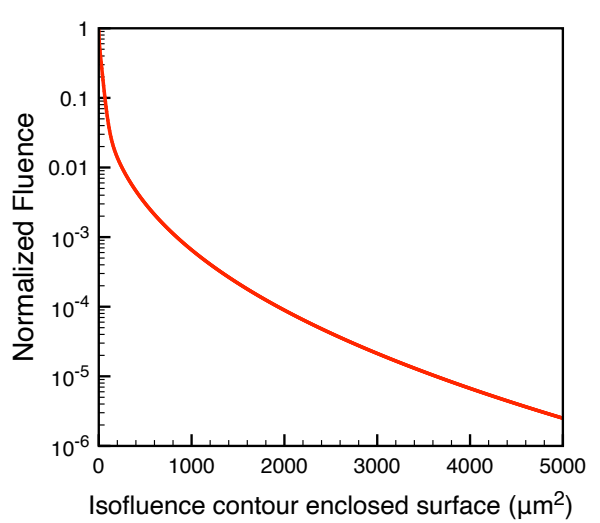

(b)

Figure 1. (a) Schematic drawing of the experimental set-up. The X-ray free electron laser (XFEL) beam impinges upon the Ge foil at a $45^{\circ}$ angle. The X-rays emitted around $30^{\circ}$ with respect to (w.r.t.) the target normal are then diffracted by a beryl crystal before reaching the CCD camera. (b) Fluence scan of the laser spot. The laser fluence is normalised to its peak, and the $x$-axis shows the area enclosed by the isofluence profiles, as detailed in Ciricosta et al. [10]. 


\section{Radiative Transfer Simulations}

Simulations were run with the one-dimensional radiative transfer code Cretin [15] to study the uniformity of the plasma conditions. These simulations served a dual purpose: firstly to identify any possible gradients and secondly to produce reliable synthetic spectra that could be directly compared with the experimental data. The one dimensional capability of the code was necessary to study the change in the plasma conditions as a function of the depth along the targets. These were modelled as planar slabs, with the number of computational nodes across them chosen so that convergence in the kinetics calculations was obtained. The radiation field from the $X$-ray laser pulse is assumed to be Gaussian in time with the profile given above. The finite bandwidth of the incident radiation was also taken into account, and also assumed to be a Gaussian distribution in frequency.

In order to replicate the experimental conditions, where the profile of the XFEL spot caused different regions of the plasma to be illuminated with a different laser intensity, simulations were run over six orders of magnitude of the laser intensity, and the time-integrated spectra were then adequately weighted according to the experimentally measured spatial distribution of the XFEL spot, following the procedure in Ciricosta et al. (2016) [10]. This allows for direct comparison of the simulated spectra with the spectra measured in the experiment.

Figure 2 shows an example of the simulated time-and-space integrated spectrum for a photon energy of the XFEL of $1400 \mathrm{eV}$ (green line). By separating the bound-bound and bound-free contributions from the spectrum (blue and red lines respectively), it can be seen that, in this case, the emission above $1400 \mathrm{eV}$ is mostly produced by bound-free transitions. The intensity of the free-free emission in this region is several orders of magnitude lower, and therefore is not shown here. Given that the bound-free spectral emission has a characteristic energy dependence given by $I \propto e^{-E / k_{B} T}$, it is possible to obtain an equivalent temperature by fitting the slope of the spectrum at these photon energies. Although this method can be used to characterise the temperature of isothermal plasmas [20], care must be taken in this case with the interpretation of the equivalent temperature, as this is an integrated measurement and the spectrum does not correspond to a single temperature.

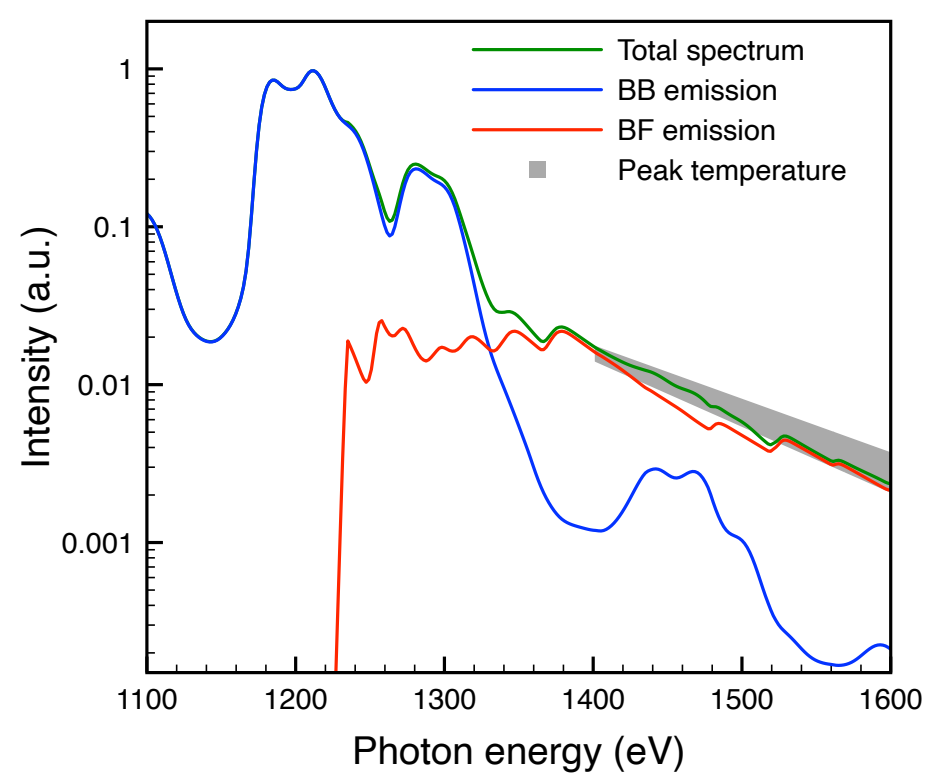

Figure 2. Bound-bound and bound-free components of the time-and-space integrated spectrum from the Cretin simulations corresponding to an XFEL photon energy of $1400 \mathrm{eV}$. For energies of the emitted photon above $1400 \mathrm{eV}$, the spectral emission is dominated by bound-free transitions. The gray area shows the slope of the bound-free emission at the peak temperature $\pm 10 \%$, which agrees with that of the integrated spectrum. 
However, in this experiment, the spectral emission of the plasma is gated by the XFEL pulse, and therefore the time-integrated spectrum is heavily weighted by the conditions at the peak of the pulse (this was confirmed by the simulations). Additionally, owing to the large effective area of the laser spot on target, the contribution to the overall spectra from the wings of the spot is negligible compared to that from the high intensity regions. These two characteristics combined cause the time-and-space integrated spectra to be representative of the plasma conditions at the peak intensity of the laser, and therefore the temperature that can be obtained from the bound-free slope is comparable to the peak temperature reached by the plasma. The Cretin simulations show that the difference between the peak temperature and that obtained from fitting the integrated spectra is below $10 \%$. This is illustrated with the gray band in Figure 2, which corresponds to the slope of the bound-free emission at the peak temperature $( \pm 10 \%)$. It can be seen that the slope of the space- and time-integrated spectrum is representative of this condition.

\section{Results}

\subsection{Simulation Results}

Figure 3 shows the results for the temperature and mean charge predicted across the target for the four XFEL photon energies of consideration. The vertical axis of the figures corresponds to the depth of the target, with the XFEL beam, irradiating at $0 \mathrm{~nm}$ (top of the figures). The pulse shape is plotted for comparison. It can be seen that, except for the case at $1400 \mathrm{eV}$, there is a significant temperature gradient across the target, with the surface that is irradiated by the laser (top) being about two times hotter than the back of the target. Surprisingly, in the $1400 \mathrm{eV}$ case, this hot region at the front of the target is not present, and the target is uniformly heated to $\sim 100 \pm 20 \mathrm{eV}$.

Similar gradients appear in the mean charge $(\bar{Z})$ of the targets. For a photon energy of $1400 \mathrm{eV}$, after the XFEL beam has left the target (around $t=250 \mathrm{fs}$ ), the ionisation is roughly constant across the depth of the plasma, and the mean charge equals 12-13. However, for the other photon energies under consideration, the hot region at the front of the target gets ionised further $(\bar{Z} \sim 16)$, whereas the back of the target remains at lower ionisation levels. This is most noticeable for a XFEL photon energy of $1300 \mathrm{eV}$, where the front of the target is S-like $(\bar{Z} \sim 16)$, while the back of the target is Sc-like $(\bar{Z} \sim 11)$.

The reason for this more uniform behaviour when the photon energy of the XFEL beam is $1400 \mathrm{eV}$ is related to both the position of bound-bound (L-M) transitions and the L-edges of the various ion stages as the plasma heats up. This can be understood by studying Figure 4, where we show results of Cretin simulations of the opacity at the front (left) and back (right) of the targets for XFEL energies of 1300 (Figure 4a) and $1400 \mathrm{eV}$ (Figure 4b) as a function of time and photon energy, for the peak intensity of the laser. In this case, the front refers to the laser-irradiated surface of the target, and the laser pulse is indicated in white in all cases. Additionally, the photon energy of the XFEL is indicated as a dashed orange line. Each of the inset plots below the figures shows a more detailed lineout of the time evolution of the plasma opacity at the photon energy of the XFEL.

It can be seen that, initially, the bound-bound absorption lines lie around $\sim 1190-1220 \mathrm{eV}$, whereas the L (bound-free) absorption edge of the cold material sits at $\sim 1250 \mathrm{eV}$. That is to say in all cases the photon energy of the XFEL lies above the cold L-edge, and thus is initially efficiently absorbed, and thus the target starts to heat and be ionised. As higher charge states are generated, both the L-M bound-bound transitions and the bound-free L-edges of these higher charge states lie at higher energies. In both the 1300 and $1400 \mathrm{eV}$ cases, the heating is such that rapidly the L-edges of the ions produced lie above the incident photon energy, and the L-shell bound-free absorption decreases (which leaves photoionisation of the $\mathrm{M}$ shell as the main laser absorption mechanism). In the case of an incident photon energy of $1300 \mathrm{eV}$, just after the middle of the XFEL pulse, the heating is such that the L-M bound-bound transitions of the ions come into resonance, and the absorbed energy increases rapidly as a consequence, and the associated shorter absorption depth leads to the large temperature gradients shown in Figure 3. In contrast, for an incident photon energy of $1400 \mathrm{eV}$, 
we have a situation where the L-M transitions never come into resonance, and the L-edges of the ions in the heated system lie above the photon energy, leaving only M-shell photoionisation as the dominant absorption mechanism throughout the laser pulse. This bound-free M-shell absorption is about an order of magnitude less than that of the L-shell, and thus the $1400 \mathrm{eV}$ photons are poorly absorbed, but the plasma as a consequence has a more uniform temperature gradient. Whilst not plotted here for reasons of space, for higher incident photon energies we can see even from these plots that they will start to lie above the L-edges of the ions in the heated system, thus again leading to increased absorption at the expense of a reduction in spatial uniformity.

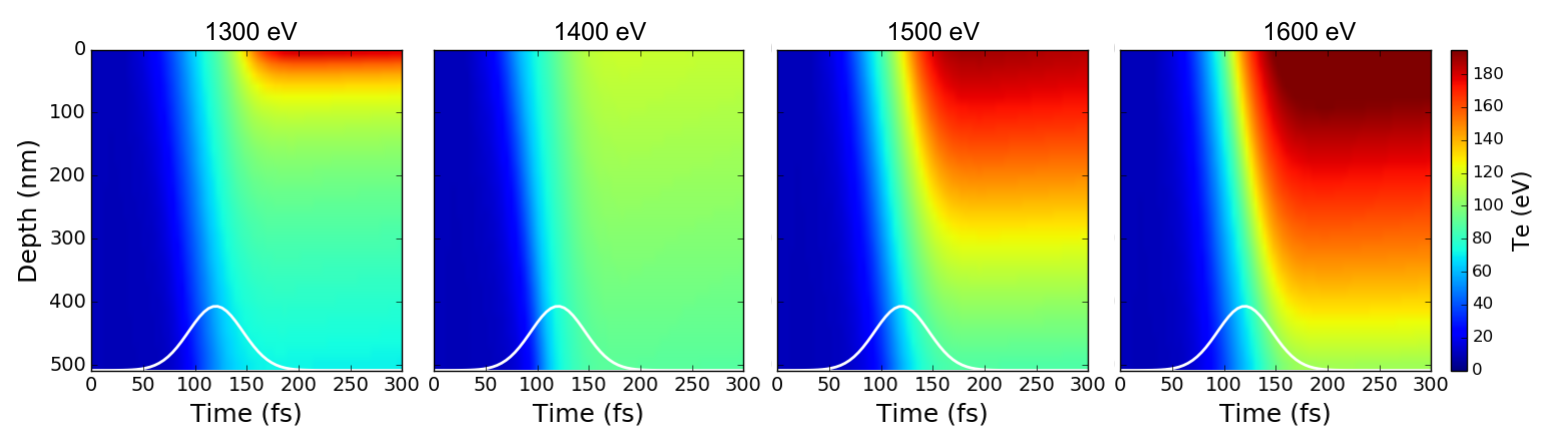

(a)
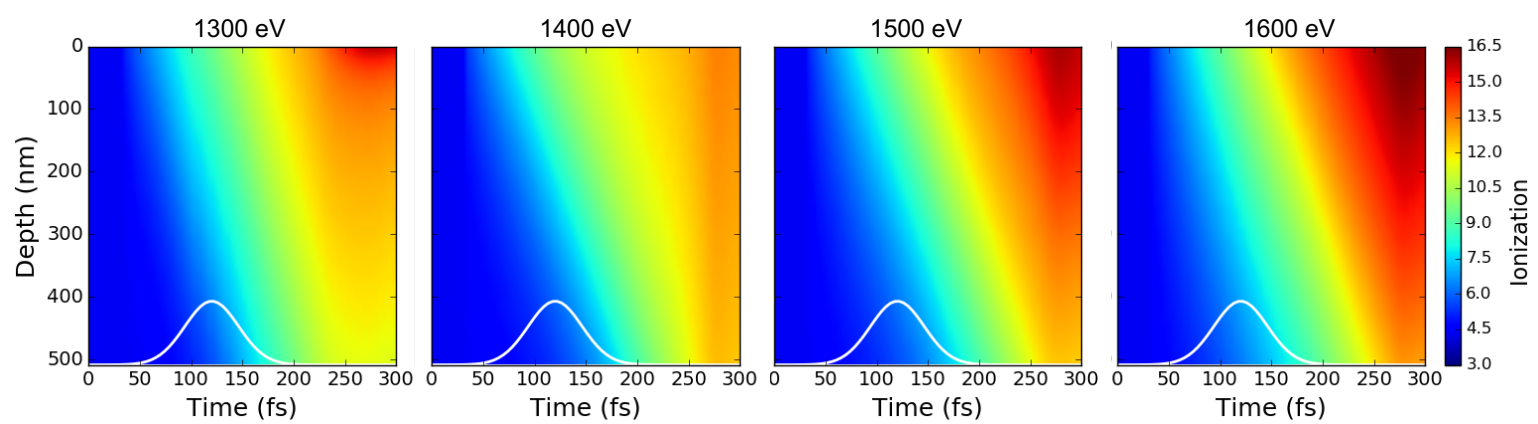

(b)

Figure 3. Cretin simulations of the temperature (a) and ionisation (b) distribution along the target as a function of time for the four photon energies of study. The laser pulse is shown in white. Note how, for the cases in which the photon energy of the laser is such that there is strong absorption $(1300,1500$ and $1600 \mathrm{eV}$ ), the front surface of the target (top region of the images) is much hotter and ionized than the back owing to the rapid absorption of the XFEL radiation before it propagates to the back of the target. However, in the $1400 \mathrm{eV}$ case, as the photon energy lies between the bound-bound (L-M) transitions and below most of the L-edges of the ions, the laser is uniformly deposited along the thickness of the target and gradients are minimised. 

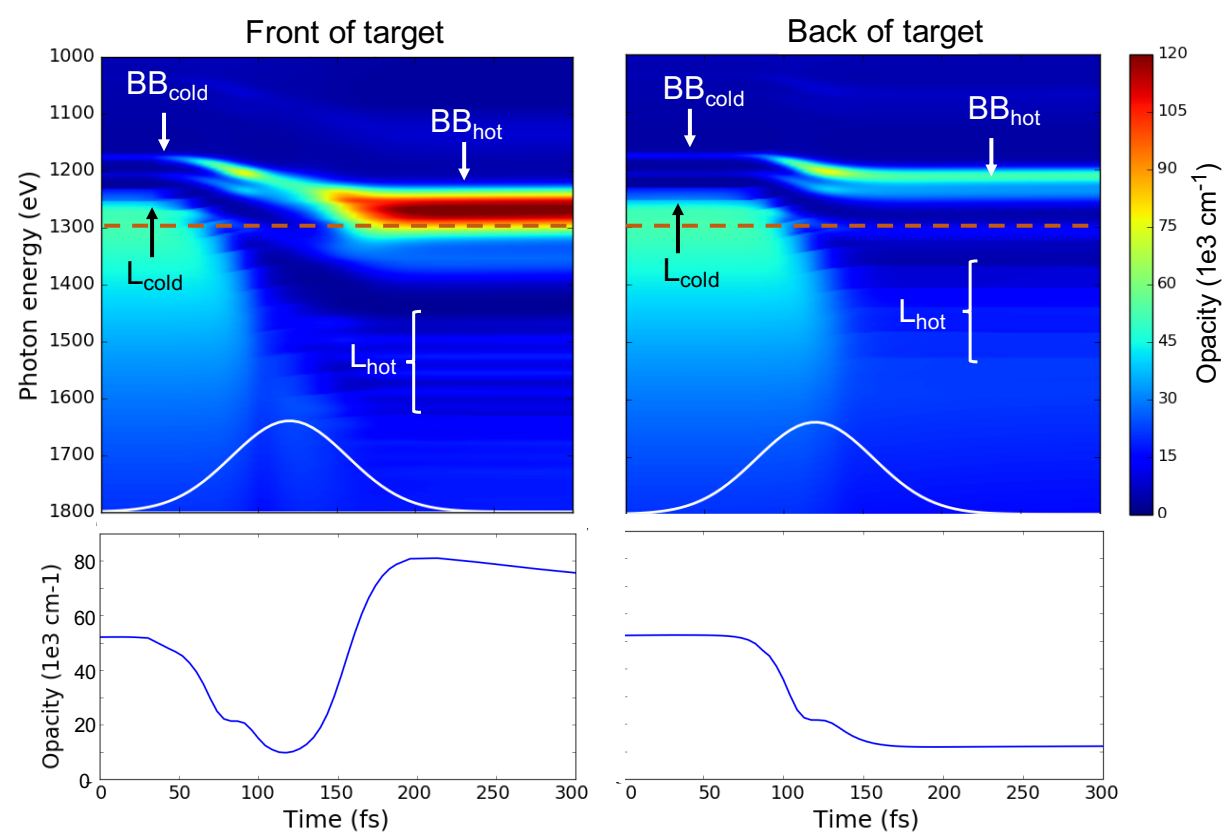

(a)
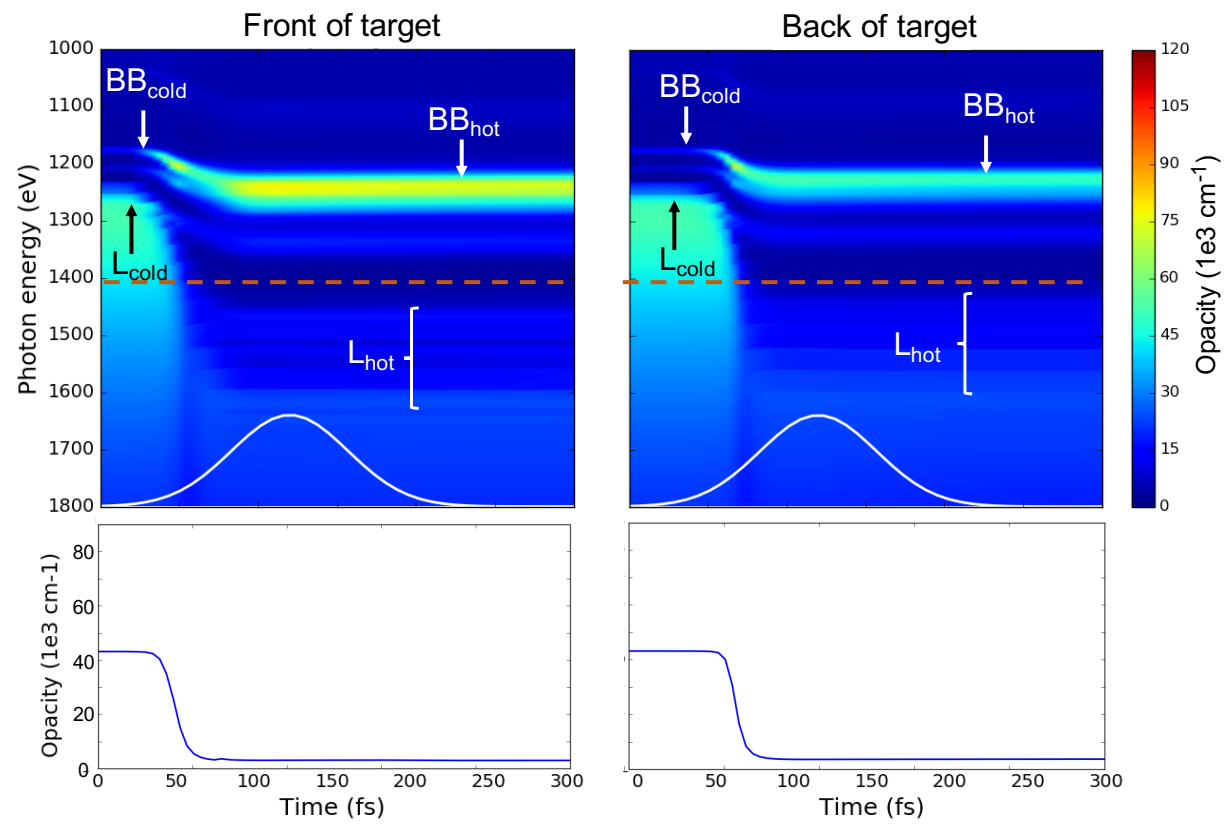

(b)

Figure 4. Cretin simulations of the opacity at the front and back of the targets for XFEL photon energies of $1300 \mathrm{eV}$ (a) and $1400 \mathrm{eV}(\mathbf{b})$. The orange dashed lines indicate the photon energy of the XFEL in each case, and the laser pulse is shown in white. Additionally, the position of the bound-bound transitions and the L-edges for the cold and hot case are indicated. Each of the inset plots below the figures show a more detailed lineout of the plasma opacity at the photon energy of the XFEL as a function of time. In the $1300 \mathrm{eV}$ case, as the plasma heats up owing to the effect of the XFEL, the bound-bound features shift up in energy until they overlap with the XFEL energy, and thus absorption becomes much more efficient. In contrast, in the $1400 \mathrm{eV}$ case, the XFEL photon energy lies above that of the bound-bound transitions, but below the L-edges of the ions produced as the plasma is heated, thus leading to reduced absorption. 


\subsection{Experimental Results}

Good quality spectra were obtained for the four XFEL photon energies of consideration. Spectra were constructed by integrating single photon detections along energy contours on the CCD image, and were then corrected for energy dispersion, solid angle projection, filter transmission and detector response. Figure 5 shows the average spectra obtained for the four XFEL photon energies of consideration, compared with the results from the radiative transfer simulations. The gray regions in the figure indicate the photon energy above which the spectral emission is dominated by bound-free transitions for each case. Note how the simulations agree with the experimental spectra in the slope of the bound-free regions, whereas the bound-bound emission features (at energies lower than those indicated with the gray regions) are not well reproduced by the simulations. This is not surprising, as modelling open shell L-shell spectra of mid- and high-Z elements is a complicated issue that the code-developing community is currently working on (see Review of the 11th non-LTE code comparison Workshop in preparation). The level of detail necessary to treat all the atomic transitions involved in these spectra requires extremely complicated atomic data, that are usually simplified, which can underestimate the contribution of some transitions to the overall spectra.

As described in the previous section, the peak temperature of the plasma $(T)$ was determined by fitting the bound-free region of each measured spectrum (right of the gray regions in Figure 5), to the function

$$
I=K+I_{0} e^{-E / k_{B} T},
$$

where $K$ is a constant introduced to account for any background emission, $I_{0}$ is a scaling parameter for the spectral intensity, $E$ is the energy of the emitted photons, and $k_{B}$ is Boltzmann's constant.

We found that variations in the XFEL pulse energy of $\pm 15 \%$, translate into significant variations in the spectral intensity. This is shown in Figure 6, where the spectra from different shots with a XFEL photon energy of $1400 \mathrm{eV}$ are shown together as a function of the pulse energy together with the average spectrum. These intensity variations arise from differences in the temperature of the emitting region of the plasma, as indicated by Equation (1), which allowed us to obtain an estimate of the error introduced in our temperature measurement by the deviations from the nominal laser energy. In Figure 7, we show the comparison between the experimentally obtained temperatures and the peak temperatures predicted by the simulations for the four photon energies of consideration. The triangles represent the peak temperature predicted by Cretin, with the error bars corresponding to variations in this peak temperature with the change of the laser energy. The circles correspond to the temperature obtained from fitting the average spectra for each photon energy, with the error bars corresponding to the standard deviation of the fit parameters. The colored rectangles represent the range of temperatures obtained from individual shots by comparing their absolute spectral bound-free intensity with that of the average spectrum (shown in Figure 6), as given by $I \propto e^{(-E / k T)}$.

Note that, while the temperature obtained from the average spectrum does not correspond in general with the average temperature, owing to the non-linearity of Equation (1), the change in slope of the bound-free emission is monotonic in temperature. Therefore, over a small range of incident intensities, as is the case in this paper, this approximation is valid. Note how, as seen in Figure 6, the slope of the bound-free emission from single shot spectra is very similar among different shots, which is indicative of small changes in the temperature. Moreover, the measured variations in the absolute intensity of the bound-free emission from single-shots, also correspond to small changes in the temperature between different shots, as shown by the colored rectangles in Figure 7.

As expected, the lowest temperature is found for the $1400 \mathrm{eV}$ case, where the laser absorption within the plasma is minimal. Additionally, as the plasma is most uniform, the temperature variations observed are minimal as well. On the contrary, for the $1300 \mathrm{eV}$ case, Cretin predicts variation of $\sim 50 \%$ in the temperature as the laser energy is modified, owing to the sensitivity of the peak temperature achieved in this case to the position of the bound-bound absorption features. Although such variation is not found in our experimental measurements, that should not be surprising, given that (as mentioned 
above), the L-shell structure of mid- and high-Z materials is extremely complicated to model, and small deviations from the real position of the lines can give rise to considerable temperature variations.

For photon energies other than $1400 \mathrm{eV}$, the large peak temperatures measured (60 to $160 \%$ greater than the peak temperature measured for $1400 \mathrm{eV}$ ) are owed to the hot layer that is generated at the front of the target, in agreement with the Cretin simulations.

Additionally, note how for the 1500 and $1600 \mathrm{eV}$ cases, the error in the temperature obtained from the average spectrum is extremely large. This can be understood by looking at Figure 5, where we show that for these cases, although the signal above $\sim 1490 \mathrm{eV}$ is mostly caused by bound-free emission, the slope is heavily affected by the multiple absorption edges from the different charge states present in the plasma, which therefore affect the fit of the slope.

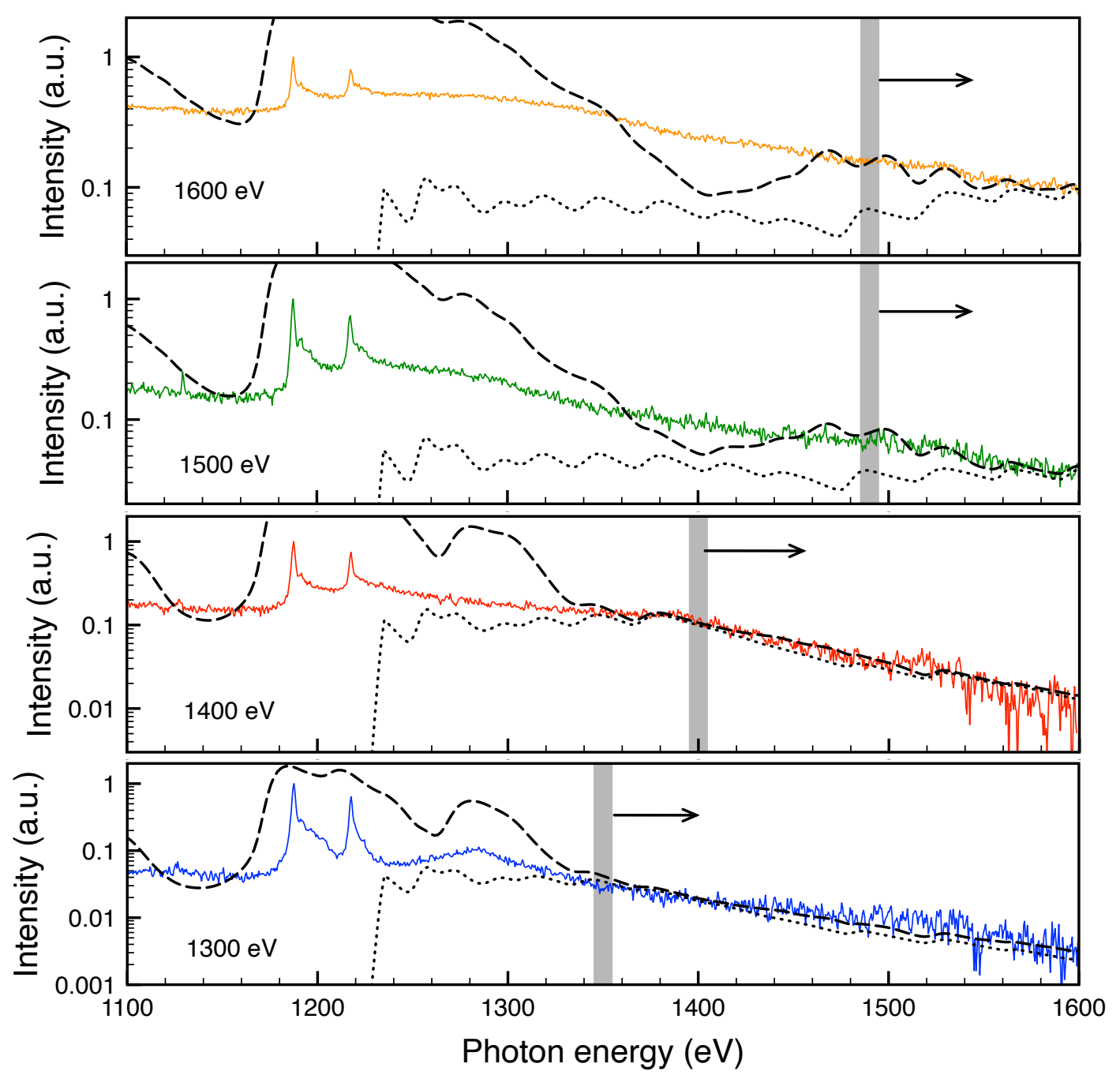

Figure 5. Comparison between the experimental (solid, colored) and simulated spectra (dashed) for the four different XFEL photon energies. The dotted lines correspond to the bound-free contribution to the overall simulated spectra. The gray area indicates the photon energy above which the emission has been used to estimate the temperature. For the 1500 and $1600 \mathrm{eV}$ cases, the bound-free emission presents several edges from the different charge states in the plasma, which makes getting a temperature from the slope more subject to error. In all cases the simulations agree with the data in the regions above the gray areas, whereas the bound-bound emission is poorly reproduced. The experimental spectra for $1300,1400,1500$ and $1600 \mathrm{eV}$ have been averaged over 102,116, 156 and 122 shots respectively. 


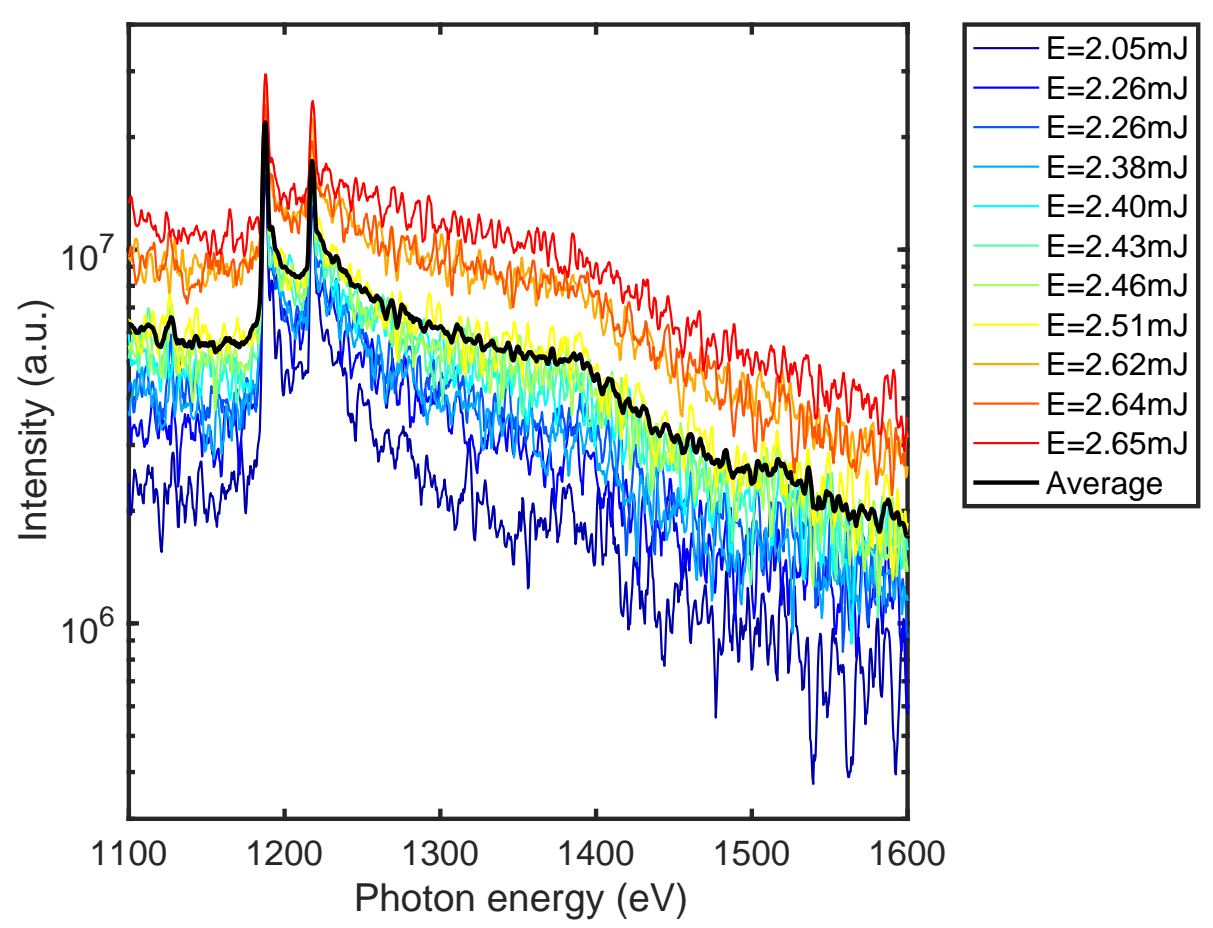

Figure 6. Spectra obtained for the different shots with a XFEL photon energy of $1400 \mathrm{eV}$ as a function of the beam energy. The small variations in the beam energy affect the spectral intensity, and effectively, the temperature of the plasma.

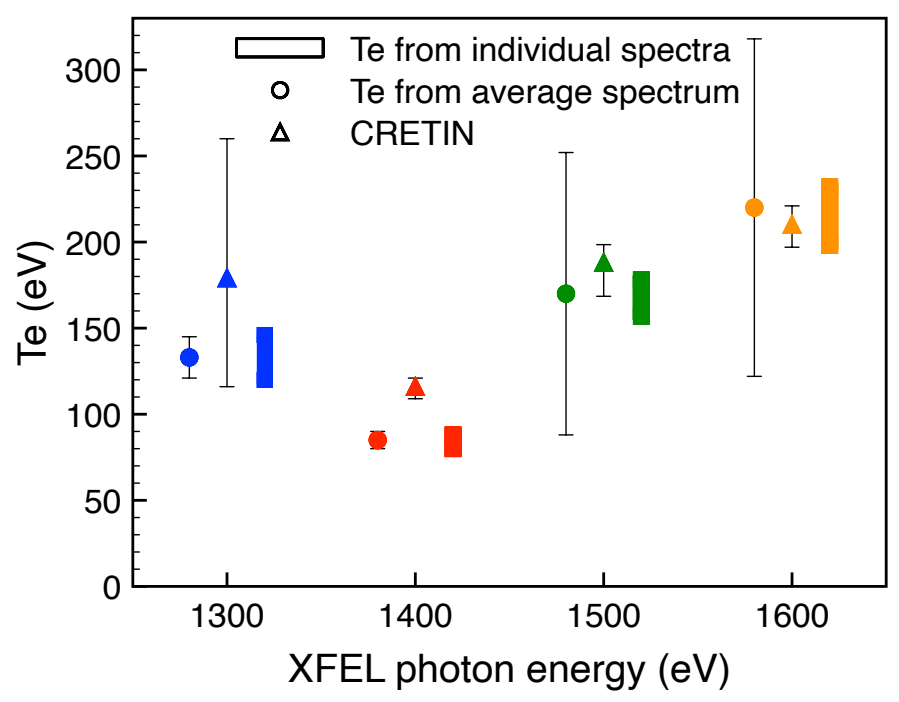

Figure 7. Comparison between the peak temperature of the plasma predicted by Cretin (triangles) and the results obtained from the experimental spectra for the different XFEL photon energies of consideration. The error bars in the Cretin results correspond to the variation of the peak temperature of the plasma when the intensity of the laser is varied by $\sim \pm 15 \%$ as in the experiment. The circles correspond to the temperatures obtained from fitting the shot-averaged spectra, whereas the colored rectangles represent the range of temperatures obtained from individual shots by comparing their absolute spectral bound-free intensity with that of the average spectrum (shown in Figure 6), as given by $I \propto e^{(-E / k T)}$. 


\section{Discussion and Conclusions}

We have presented the results from a experiment at LCLS in which solid-density germanium plasmas were generated using four different photon energies. We have shown that careful consideration of the photon energy of the XFEL with respect to the position of the edges of the dominant ionization stages and bound-bound transitions in a heated plasma is necessary to achieve uniformity in the plasma conditions.

The experimental spectra have been modeled using one-dimensional radiative transfer simulations, weighted accordingly with the measured profile of the laser spot. The synthetic spectra showed that the time-and-space integrated spectra are heavily weighted by the plasma conditions near the peak intensity of the laser pulse, and are therefore a reasonable diagnostic of the peak temperature of the plasma. This temperature can be extracted from the slope of the bound-free spectral emission which allowed us to compare the experimental results with the predictions from the simulations.

We observed that the peak temperature of the plasma is lowest for a XFEL photon energy of $1400 \mathrm{eV}$, as this energy lies between bound-bound and bound-free absorption features in hot germanium, and therefore no hot layer is generated at the front of the target. The agreement between experiment and simulations provides further confidence that the generated conditions for this case are uniform across the target. Our measurements also show how for lower $(1300 \mathrm{eV})$ and higher (1500 and $1600 \mathrm{eV}$ ) XFEL photon energies, the obtained peak temperatures are larger, owing to resonance with the L-M bound-bound transitions, and lying above the L-edge of the ions in the heated system respectively. Thus, the main features of the spectra, in terms of the temperatures extracted from them, are broadly in good agreement with the computational results.

This work shows that generating uniform conditions in solid density plasmas created with a XFEL for high-Z materials requires careful consideration of the experimental parameters, as the complicated atomic structure of these elements greatly complicates the interaction between the XFEL and the plasma itself.

Author Contributions: Conceptualization, S.M.V., T.R.P. and J.S.W.; Investigation, S.M.V., T.R.P., B.A.H., H.-K.C., T.B., V.V., M.-F.L., T.B.v.D. and J.S.W.; Formal analysis, G.P.-C., S.R. and R.R.; Software, G.P.-C., S.R., O.H., R.R.; Funding acquisition, S.M.V. and J.S.W.; Project administration, S.M.V. and J.S.W.; writing—original draft preparation, G.P.-C.; writing-review and editing, S.M.V. and J.S.W. All authors have read and agreed to the published version of the manuscript.

Funding: Use of the Linac Coherent Light Source (LCLS), SLAC National Accelerator Laboratory, is supported by the U.S. Department of Energy, Office of Science, Office of Basic Energy Sciences under Contract No. DE-AC02-76SF00515. The SXR Instrument is funded by a consortium whose membership includes the LCLS, Stanford University through the Stanford Institute for Materials Energy Sciences (SIMES), Lawrence Berkeley National Laboratory (LBNL), University of Hamburg through the BMBF priority program FSP 301, and the Center for Free Electron Laser Science (CFEL). G.P.-C., and J.S.W. gratefully acknowledge support from LLNL under grant number B617350. This work was performed under the auspices of the U.S. Department of Energy by Lawrence Livermore National Laboratory under Contract DE-AC52-07NA27344. S.M.V. is a Royal Society University Research Fellow. S.M.V., S.R., M.F.K., R.R. and J.S.W. acknowledge support from the U.K. EPSRC under grant EP/P015794/1. O.H. acknowledges support from the Oxford University Centre for High Energy Density Physics (OxCHEDS). This document was prepared as an account of work sponsored by an agency of the United States government. Neither the United States government nor Lawrence Livermore National Security, LLC, nor any of their employees makes any warranty, expressed or implied, or assumes any legal liability or responsibility for the accuracy, completeness, or usefulness of any information, apparatus, product, or process disclosed, or represents that its use would not infringe privately owned rights. Reference herein to any specific commercial product, process, or service by trade name, trademark, manufacturer, or otherwise does not necessarily constitute or imply its endorsement, recommendation, or favoring by the United States government or Lawrence Livermore National Security, LLC. The views and opinions of authors expressed herein do not necessarily state or reflect those of the United States government or Lawrence Livermore National Security, LLC, and shall not be used for advertising or product endorsement purposes. The Czech co-authors appreciate a financial support provided by the Czech Science Foundation (project GA20-08452S) and the Czech Ministry of Education, Youth and Sports (projects LTT17015 and EF16_013/0001552).

Conflicts of Interest: The authors declare no conflict of interest. 


\section{References}

1. Vinko, S.M.; Ciricosta, O.; Cho, B.I.; Engelhorn, K.; Chung, H.K.; Brown, C.R.D.; Burian, T.; Chalupsky, J.; Falcone, R.W.; Graves, C.; et al. Creation and diagnosis of a solid-density plasma with an X-ray free-electron laser. Nature 2012, 482, 59-62. [CrossRef] [PubMed]

2. Cho, B.I.; Engelhorn, K.; Vinko, S.M.; Chung, H.K.; Ciricosta, O.; Rackstraw, D.S.; Falcone, R.W.; Brown, C.R.D.; Burian, T.; Chalupský, J.; et al. Resonant $K \alpha$ Spectroscopy of Solid-Density Aluminum Plasmas. Phys. Rev. Lett. 2012, 109, 245003. [CrossRef] [PubMed]

3. Ciricosta, O.; Vinko, S.M.; Chung, H.K.; Cho, B.I.; Brown, C.R.D.; Burian, T.; Chalupský, J.; Engelhorn, K.; Falcone, R.W.; Graves, C.; et al. Direct Measurements of the Ionization Potential Depression in a Dense Plasma. Phys. Rev. Lett. 2012, 109, 065002. [CrossRef] [PubMed]

4. Ciricosta, O.; Vinko, S.M.; Barbrel, B.; Rackstraw, D.S.; Preston, T.R.; Burian, T.; Chalupský, J.; Cho, B.I.; Chung, H.K.; Dakovski, G.L.; et al. Measurements of continuum lowering in solid-density plasmas created from elements and compounds. Nat. Commun. 2016, 7, 1-6. [CrossRef]

5. Vinko, S.M.; Ciricosta, O.; Wark, J.S. Density functional theory calculations of continuum lowering in strongly coupled plasmas. Nat. Commun. 2014, 5, 1-7. [CrossRef] [PubMed]

6. Vinko, S.M.; Ciricosta, O.; Preston, T.R.; Rackstraw, D.S.; Brown, C.R.D.; Burian, T.; Chalupský, J.; Cho, B.I.; Chung, H.K.; Engelhorn, K.; et al. Investigation of femtosecond collisional ionization rates in a solid-density aluminium plasma. Nat. Commun. 2015, 6, 6397. [CrossRef] [PubMed]

7. $\quad$ van den Berg, Q.Y.; Fernandez-Tello, E.V.; Burian, T.; Chalupský, J.; Chung, H.K.; Ciricosta, O.; Dakovski, G.L.; Hájková, V.; Hollebon, P.; Juha, L.; et al. Clocking Femtosecond Collisional Dynamics via Resonant X-ray Spectroscopy. Phys. Rev. Lett. 2018, 120, 055002. [CrossRef] [PubMed]

8. Preston, T.R.; Vinko, S.M.; Ciricosta, O.; Hollebon, P.; Chung, H.K.; Dakovski, G.L.; Krzywinski, J.; Minitti, M.; Burian, T.; Chalupský, J.; et al. Measurements of the K-Shell Opacity of a Solid-Density Magnesium Plasma Heated by an X-ray Free-Electron Laser. Phys. Rev. Lett. 2017, 119, 085001. [CrossRef] [PubMed]

9. Rackstraw, D.S.; Ciricosta, O.; Vinko, S.M.; Barbrel, B.; Burian, T.; Chalupský, J.; Cho, B.I.; Chung, H.K.; Dakovski, G.L.; Engelhorn, K.; et al. Saturable Absorption of an X-ray Free-Electron-Laser Heated Solid-Density Aluminum Plasma. Phys. Rev. Lett. 2015, 114, 015003. [CrossRef] [PubMed]

10. Ciricosta, O.; Vinko, S.M.; Chung, H.K.; Jackson, C.; Lee, R.W.; Preston, T.R.; Rackstraw, D.S.; Wark, J.S. Detailed model for hot-dense aluminum plasmas generated by an X-ray free electron laser. Phys. Plasmas 2016, 23, 022707. [CrossRef]

11. Chung, H.K.; Chen, M.; Morgan, W.; Ralchenko, Y.; Lee, R. FLYCHK: Generalized population kinetics and spectral model for rapid spectroscopic analysis for all elements. High Energy Density Phys. 2005, 1, 3-12. [CrossRef]

12. Chung, H.K.; Chen, M.; Lee, R. Extension of atomic configuration sets of the Non-LTE model in the application to the $\mathrm{K} \hat{\mathrm{I}} \pm$ diagnostics of hot dense matter. High Energy Density Phys. 2007, 3, 57-64. [CrossRef]

13. Bar-Shalom, A.; Oreg, J.; Goldstein, W.H.; Shvarts, D.; Zigler, A. Super-transition-arrays: A model for the spectral analysis of hot, dense plasma. Phys. Rev. A 1989, 40, 3183-3193. [CrossRef] [PubMed]

14. Chen, M.H.; Crasemann, B.; Mark, H. Relativistic K-shell Auger rates, level widths, and fluorescence yields. Phys. Rev. A 1980, 21, 436-441. [CrossRef]

15. Scott, H.A. CRETIN-A radiative transfer capability for laboratory plasmas. J. Quant. Spectrosc. Radiat. Transfer 2001, 71, 689-701. [CrossRef]

16. Schlotter, W.F.; Turner, J.J.; Rowen, M.; Heimann, P.; Holmes, M.; Krupin, O.; Messerschmidt, M.; Moeller, S.; Krzywinski, J.; Soufli, R.; et al. The soft X-ray instrument for materials studies at the linac coherent light source X-ray free-electron laser. Rev. Sci. Instrum. 2012, 83, 043107. [CrossRef] [PubMed]

17. Düsterer, S.; Radcliffe, P.; Bostedt, C.; Bozek, J.; Cavalieri, A.; Coffee, R.; Costello, J.T.; Cubaynes, D.; DiMauro, L.; Ding, Y.; et al. Femtosecond X-ray pulse length characterization at the Linac Coherent Light Source free-electron laser. New J. Phys. 2011, 13, 093024. [CrossRef]

18. Sorokin, A.; Jastrow, U.; Juranic, P.; Kapitzki, S.; Tiedtke, K.; Richter, M.; Arp, U.; Moeller, S.; Turner, J.; Schlotter, W. Report on pulse energy monitoring at the SXR beamline using gas-monitor detectors. Tech. Rep. SLAC 2010. 
19. Chalupskỳ, J.; Krzywinski, J.; Juha, L.; Hájková, V.; Cihelka, J.; Burian, T.; Vyšín, L.; Gaudin, J.; Gleeson, A.; Jurek, M.; et al. Spot size characterization of focused non-Gaussian X-ray laser beams. Opt. Express 2010, 18, 27836-27845. [CrossRef] [PubMed]

20. Kilkenny, J.D.; Lee, R.W.; Key, M.H.; Lunney, J.G. X-ray spectroscopic diagnosis of laser-produced plasmas, with emphasis on line broadening. Phys. Rev. A 1980, 22, 2746-2760. [CrossRef]

Publisher's Note: MDPI stays neutral with regard to jurisdictional claims in published maps and institutional affiliations.

(C) 2020 by the authors. Licensee MDPI, Basel, Switzerland. This article is an open access article distributed under the terms and conditions of the Creative Commons Attribution (CC BY) license (http:// creativecommons.org/licenses/by/4.0/). 